DOI: $10.1515 /$ ausp-2015-0030

\title{
Die Rolle der Prestige im Sprachkontakt
}

\author{
Attila KELEMEN \\ Lehrstuhl für Angewandte Sprachwissenschaft \\ Sapientia Universität, Fakultät für Technische- und Geisteswissenschaften \\ akelemen20@yahoo.no
}

\begin{abstract}
Abstrakt. Die vorliegende Arbeit behandelt die Rolle des äußeren Faktors „Prestige“ im Kontakt zwischen Sprachen, die Art und Weise, wie das Ansehen, das Dominanzverhältnis die Entfaltung und die Richtung des Entlehnungsprozesses, sowie das Überleben gewisser Wörter und Strukturen und sogar das Schicksal einer Sprache entscheiden.
\end{abstract}

Schlüsselwörter: Prestige, Dominanzverhältnis, Entlehnung, Sprachkontakt, Sprecher

\begin{abstract}
This paper deals with the role of the external factor "prestige' in the contact between languages, the way how the prestige, the dominance relationship decides the development and direction of the borrowing process, as well as the survival of certain words and structure, and even the fate of a language.
\end{abstract}

Keywords: prestige, dominance relationship, borrowing, language contact, speakers

Die Sprachen ändern sich im Laufe der Zeit, und das geschieht aus mehreren Gründen. Öfters ergeben sich die sprachlichen Änderungen und Variationen aus der Zusammenwirkung verschiedener Faktoren, die traditionell in zwei Typen eingeteilt werden: interne, innere oder innersprachliche Faktoren, beziehungsweise externe, äußere oder außersprachliche Faktoren.

Unter inneren Faktoren verstehen wir diejenigen Faktoren der sprachlichen Änderungen, die inhärent sind, die sich im Inneren der Sprache befinden und im Sprachsystem in irgendwelchem synchronischen Stadium vorkommen können. Diese Faktoren machen eigentlich die existierenden phonetischen, morphologischen und syntaktischen Voraussetzungen aus. Die innere Struktur der Sprachen ist dynamisch und kann sich als Folge der Aktion natürlicher inhärenter Kräfte ändern. Sapir (1970 [1921]) ist der Meinung, die Sprache habe eine Drift, eine inhärente Treibkraft: „Language moves down time in a current of its own making. 
It has a drift.“ (Die Sprache ändert sich im Laufe der Zeit in die Richtung ihres eigenen Werdens. Sie hat eine Drift.)

Wenn sich eine sprachliche Änderung als eine innere Entwicklung erweist, dann ist es wahrscheinlich, dass diese in allen Sprachen stattgefunden hat, weil das innere System der Sprachen so etwas aus irgendeinem Grund verlangt. Es gibt zwei Kategorien von inneren Faktoren: strukturelle Modelle und typologische Einschränkungen.

Fennell (2001) spricht über innere Faktoren, wobei die Motivationen die folgenden sind: die Leichtigkeit der Artikulation (X ist leichter auszusprechen als Y), die Analogie (die Anwendung eines Phänomens für andere Phänomene durch Assoziation), die Reanalyse, der Zufall (laut Crystall 1987: 333). Andere oft erwähnte innere Ursachen der sprachlichen Änderungen sind Faktoren, wie die Vorliebe des Sprechers für einfache und durchsichtige Systeme, welche die Sprachlernenden dazu bewegen können, die unregelmäßigen, undurchsichtigen Paradigmen umzugestalten.

Wenn die Änderungen von äußeren Faktoren verursacht werden, dann sind sie von einem System von außen ausgelöst, und auf diese Weise unabhängig vom inneren Mechanismus der Sprache. Unter äußeren Faktoren versteht man also diejenigen Kräfte, die aus der Lokalisierung und dem Gebrauch der Sprache in der Gesellschaft entstehen.

Die durch die Aktion äußerer Faktoren verursachten sprachlichen Änderungen können laut Fennell (2001) stattfinden infolge: der Geographie (die Absonderung einer Sprache oder Varietät von der anderen und als Alternative die Annäherung d. h. der Sprachkontakt: eine Ursache der Änderungen in einer Sprache ist die Tatsache, dass die Sprecher mit einer oder mehreren Sprachen in Kontakt kommen), des Kontakts mit neuen oder alten Phänomenen (die Notwendigkeit der Anpassung an neue, verschiedene und fortlaufende Aspekte der Gesellschaft), des unvollkommenen Erlernens (die Sprachen können sich ändern auch weil die Menschen sie nicht vollkommen erlernen: wenn die Menschen mit einer Sprache in Kontakt kommen, können sie sie imperfekt lernen, und letzten Endes sie auch ändern, indem sie den Substrat-Effekt auf die Sprache bewirken), des SubstratEffekts, des Faktors der sozialen Prestige (der Versuch eines Teiles der Sprecher, solche linguistische Eigenschaften, die als „besser” gelten und betrachtet werden als die eigenen, nachzuahmen oder sich anzueignen).

Es gibt verschiedene Theorien und Modelle bezüglich der Art und Weise, wie diese Faktoren wirken, und die Wahl der einzelnen Theorien und Modelle bestimmt auch die Perspektive der Problembehandlung. In der Fachliteratur werden die inneren Faktoren in einem generativen Rahmen behandelt, während die äußeren Faktoren in einem soziolinguistischen. Die Soziolinguistik operiert mit Begriffen wie Determinanten und Determinantenverhältnis. In einem soziolinguistischen Zusammenhang muss man als Verhältnis der Determinanten die 
Bedingungen oder Faktoren in einem weiteren Sinne verstehen, diejenigen Bedingungen oder Faktoren, die imstande sind, Änderungen und Variation zu verursachen. „Determinante“ oder „Bestimmungsfaktor“ bedeutet ein Prinzip, ein Rahmen, der auf die Sprache auswirkt. In der Soziolinguistik wird gewöhnlich nicht empfohlen, einen Einzelfaktor als einzige Ursache einer gewissen linguistischen Änderung anzugeben, weil es in den meisten Fällen mehrere verschiedene Faktoren gibt, die zusammen wirken.

Man muss wissen, dass eine Reihe von Entwicklungen und Änderungen, vor allem die syntaktischen, von der Wechselwirkung etlicher inneren und äußeren Faktoren verursacht werden können. Während der Unterschied zwischen inneren und äußeren Ursachen in einigen Fällen sehr klar erscheint, ist die Absonderung dieser Faktoren nicht immer gerechtfertigt oder sogar gewünscht, insbesondere im Bereich der syntaktischen Änderung, meint Mithun im Artikel External triggers and internal guidance in syntactic development: Coordinating conjunction. Andere behaupten, dass die inneren und äußeren Faktoren voneinander unterschieden und getrennt werden müssen. Manchmal ist es schwer, die inneren und äußeren Faktoren voneinander abzugrenzen, weil die syntaktische Änderung oftmals das Ergebnis der Interaktion dieser Faktoren ist.

Die häufigste äußere Ursache der Änderungen, die in einer Sprache vorkommen, ist der Sprachkontakt, und folglich die Entlehnung. Sprachen können aus verschiedenen Gründen in Kontakt kommen. Der Kontakt zwischen zwei Sprachen hat als Ergebnis verschiedene sprachliche und soziolinguistische Phänomene und setzt in Bewegung verschiedene Mechanismen des Sprachwandels / der sprachlichen Änderungen. McMahon (1992: 209) behauptet, indem er Winter (1973: 144) zitiert, dass kein Bestandteil der natürlichen Sprache immun sei gegenüber den Änderungen, die unter dem Einfluss der Sprachen von draußen entstehen.

Samuels (1972: 92) ist der Meinung, dass das Studium des Kontakts zwischen Sprachen verschiedener Art wichtig sei, denn es kann diejenigen Ursachen (oft die einfachsten und die unmittelbarsten) hervorheben, die bewirken, dass sich eine Sprache ändert: höchstwahrscheinlich wird sie von einer Sprache anderer Art beeinflusst als Ergebnis des Kontakts zwischen den eigenen Sprechern und denjenigen, die die andere Sprache verwenden.

Trudgill (1994) unterscheidet zwischen Sprachkontakt und Dialektkontakt, aber weist darauf hin, dass die Entlehnung und die Vereinfachung sowohl im Falle eines Sprachkontakts als auch im Falle des Dialektkontakts erscheinen. Die Entlehnung ist der Beweis des Einflusses, den eine Sprache auf die andere ausübt. Hock (1992) betrachtet die Benennung „Entlehnung“ als unpassend, da die entlehnten Wörter und Strukturen nur selten zurückgegeben werden. Haugen (1950) definiert den Entlehnungsprozess als eine absichtliche Wiedergabe oder Reproduktion der in einer Sprache vorher gefundenen Strukturen in eine andere 
Sprache. Jeder Sprecher versucht gelernte sprachliche Strukturen zu reproduzieren um sich in den neuen Sprachsituationen zurechtzufinden. Unter den neuen Strukturen, die er erlernen muss, befinden sich einige, die von seiner Muttersprache verschieden sind, und es mag sein, dass er diese wiedergeben / reproduzieren möchte. Wenn er diese reproduziert, aber nicht in jenem Zusammenhang, in dem er sie gelernt hat, sondern in einer anderen Sprache, dann kann man sagen, dass er sie aus seiner Sprache entlehnte in die andere.

Stang (1970: 28) meint, dass alle Arten von Entlehnungen aus seiner Fremdsprache - seien diese lebendig oder tot - von zwei Voraussetzungen abhängen müssen: zuerst muss jemand der Erfahrung einer Fremdsprache ausgesetzt werden, dann muss man die Rolle eines fremden Elements in seiner eigenen Muttersprache sehen. Strang verwendet die Benennung „,innere Entlehnung“ für die Entlehnung zwischen Varietäten derselben Sprache.

Weinreich ist der Meinung, dass es sehr wichtig ist, zwischen zwei Phasen oder Etappen der Interferenzerscheinung zu unterscheiden: Interferenz in der Rede (parole) und Interferenz in der Sprache (langue), die eigentlich die Entlehnung ist. Weinreich verwendet in beiden Fällen die Bezeichnung „Interferenz“. Die eigentliche Interferenz findet in der Rede / im Sprechen der Zweisprachigen statt. Wenn das Phänomen zur Gewohnheit wird, kann es auch zur Entlehnung werden, wenn es in der Sprache akzeptiert wird. In der Sprache hängt sein Gebrauch nicht mehr vom Bilingualismus ab (laut Weinreich 1953).

Die Entlehnungen können je nach dem Niveau der Sprache, wo sie stattfinden, als phonologische, morphologische, syntaktische und lexikalische Entlehnungen klassifiziert werden. Wenn der Sprachkontakt intensiver ist, kann er auf das phonemische System auswirken. Unter Interferenz im phonetischem Bereich verstehen wir jenen Vorgang, wo man die Wiedergabe eines Lautes aus seiner Sprache mit Hilfe eines Lautes der Primärsprache/Erstsprache versucht. Die Interferenz entsteht dann, wenn ein Zweisprachiger ein Phonem aus dem sekundärem System mit einem in dem primären System identifiziert, und indem er es reproduziert/wiedergibt, unterwirft er es den phonetischen Regeln der Erstsprache (laut Weinreich 1953: 14).

Die grammatische Entlehnung bildet ein umstrittenes Problem, und ist nach der Meinung Weinreichs (1953: 29) von „einer betrachtlichen Komplexität“. Die grammatische Entlehnung wird als eine Eingliederung von fremden Regeln in die Sprache betrachtet. Laut Appel und Muysken (1987: 154) gibt es mindestens fünf Möglichkeiten, in der die grammatische Entlehnung stattfinden kann: (1) durch Konvergenz, (2) durch kulturellen Einfluss und lexikalische Entlehnung, (3) durch das Erlernen einer Fremdsprache, (4) durch Relexifikation, (5) durch Nachahmung der Prestigestrukturen.

Während die Wörter aus einer Sprache in die andere leicht übergehen können, werden die Flexionsendungen selten übertragen. Inwiefern der Sprachkontakt 
morphologische Entlehnung ergibt oder nicht, hängt vom Assimilationsgrad der entlehnten Wörter ab. Im Allgemeinen nimmt man an, dass die grammatische Entlehnung möglich ist, aber gebundene Morpheme, wie z. B. Flexionsendungen, werden nur sehr selten entlehnt. Wenn die Entlehnung jedoch stattfindet, ist es der Tatsache zu verdanken, dass die Donorsprache und die Empfängersprache gewissermaßen ähnlich sind. Es scheint, dass die morphologische Entlehnung von derjenigen Sprache, die weniger gebundene Morpheme hat, ausgeht, und begibt sich in die Richtung des Systems, das mehr gebundene Morpheme verwendet. Wichtig ist auch die Entlehnung von Satzbau- und Wortbildungselementen.

In der Interferenz von zwei grammatischen Strukturen dient gewöhnlich diejenige als Vorbild, die relativ freie und unveränderliche Morpheme in ihrem Paradigma verwendet, also diejenige, die eine explizitere Struktur hat. Die syntaktische Entlehnung, die Entlehnung von grammatischen Verhältnissen wird als noch ungewöhnlicher als die morphologische. Die Interferenz in grammatischen Verhältnissen, z. B. der Wortstellungsfehler, ist sehr häufig bei Zweisprachigen, die die Zweitsprache nicht gut beherrschen.

Die häufigsten Entlehnungen sind aber die lexikalischen. Betrachtet als viel lockerer strukturiert als die Phonemik und die Grammatik, ist die Lexik, der Wortschatz einer Sprache, der bevorzugte Bereich der Entlehnung. Meillet ist der Meinung, dass es leicht ist, Wörter zu entlehnen, da die Lexik kein System ist; die Entlehnung ist aber schwierig in einem geschlossenen System, wie die Phonologie und die Grammatik.

Von den Wortarten wird das Substantiv am leichtesten entlehnt, dann Verben und Adjektive, wogegen am schwierigsten Pronomen und Artikel, laut einer Studie von Haugen (1950). Das geschieht dank der Grammatikalisierung dieser Wortklassen. Jespersen behauptet, dass die meisten Lehnwörter Substantive und Adjektive sind, während die sogenannten Funktionswörter sehr selten entlehnt werden (laut Jespersen 1922: 212).

Nicht alle Sprachkontakte sind gleich, folglich auch ihre Folgen nicht. Einige Kontaktsituationen sind zufällig und können als Ergebnis die Entlehnung einiger Wörter haben, während ein intensiverer Kontakt zu einer massiven Entlehnung von Wörtern führen kann, sowie zum Einfluss auf die grammatische Struktur. Thomason und Kaufman (1988) haben eine Skala der Entlehnungen ausgearbeitet, um den Interferenzgrad der sich im Kontakt befindenden Systeme zu messen. Ihre Skala hat folgende Niveaus entsprechend der Art des Kontakts: zufälliger Kontakt, nur lexikalische Entlehnung, die Struktur der Sprache wird nicht berührt; etwas intensiverer Kontakt, geringe strukturelle Entlehnung, es werden Konjunktionen und Adverbialpartikeln entlehnt, es erscheinen neue Phoneme in den entlehnten Wörtern; intensiverer Kontakt, etwas mehr strukturelle Entlehnungen, es werden Adpositionen (Präpositionen und Postpositionen), Personalpronomina und Demonstrativpronomina entlehnt; Phonemisierung der allophonischen Ab- 
wechslungen, Betonungsregeln usw.; starker kultureller Druck, mäßige strukturelle Entlehnung, entlehnte Affixe und morphologische Kategorien, Änderung der Wortstellung usw.; sehr starker kultureller Druck, Änderung der Typologie, phonetische Änderungen, radikale morpho-syntaktische Änderungen;

Die Prestige wird neben der Notwendigkeit als die wichtigste Ursache der Entlehnung betrachtet. McMahon (1994: 204) meint: „Einige Wörter neigen mehr zur Entlehnung (»borrowable«) als andere: insbesondere der Grundwortschatz (Wörter für die Körperteile, das Wetter, allgemeine Erfahrungen, wie Geburt und Tod, Naturphänomene, Flüsse und Berge, kleine Zahlwörter) wird nur selten berührt, und beinahe ausschließlich in Situationen, wo keine der einbezogenen Sprachen als angesehener (mit mehr Prestige) betrachtet wird als die andere.“

Es gibt zwei Grundmotivationen für die Entlehnung: die Prestige und die Notwendigkeit. Die Prestige, das Ansehen ist eng verbunden mit der Einstellung der Sprecher: die Einstellung der Entlehner gegenüber der Donorsprache und gegenüber deren Sprechern kann die Entlehnung erleichtern oder verhindern. So wirkt die Prestige dann, wenn die fremde Sprache und Kultur gerät mehr geschätzt zu werden als die eigene Sprache und Kultur. Bezüglich der Rolle der Prestige im Entlehnungsprozess sagt McMahon (1994: 224): „Sprachen mit beinahe gleichem Ansehen sind geneigt, gegenseitigen Einfluss aufzuweisen, während eine Sprache mit weniger Prestige von einer Sprache mit mehr Prestige eher entlehnen wird als umgekehrt“, und sie behauptet auch: „In solchen Situationen der ungleichen Prestige gehen die Entlehnungen von der Sprache mit mehr Prestige zu der mit weniger Prestige über, und sie werden in jenen semantischen Bereichen konzentriert, wo die Sprecher der Sprache mit mehr Prestige den größten Einfluss ausüben." (McMahon 1994: 202) Die Notwendigkeit wirkt hingegen in solchen Fällen, wo ein Volk keine entsprechenden Benennungen hat für die neuen Sachen und Phänomene, die es kennenlernt im Zusammentreffen mit einer anderen Kultur, und fühlt den akuten Bedarf, diesen Sachen und Erscheinungen einen Namen zu geben, diese in seiner eigenen Muttersprache zu benennen. In Weinreichs (1953) Terminologie erscheint diese Ursache als sprachliche Neuerung.

Nach dem relativen Status der Sprachen im Kontakt können wir über Adstratum, Substratum und Superstratum sprechen. Hock weist auf drei Modelle der Kontaktsituation (mit dem Altskandinavischen, dem Franzözisch-Normannischen und den indischen Sprachen) hin, in die die englische Sprache nacheinander einbezogen wurde. In Hocks (1991: 411) Auffassung kann man im Falle vom Altenglischen und Altskandinavischen von Adstrata sprechen, da die beiden Sprachen gleiche Prestige hatten. Sie wurden von denselben sozialen Schichten, von gleichberechtigten gesprochen. Die adstratalen Verhältnisse begünstigen die Entlehnung von Wörtern aus der Alltagssprache, sogar aus dem Grundwortschatz. Wenn die Donorsprache Superstratum ist, wie im Falle des Kontakts des Altenglischen mit dem Französisch-Normannischen, dann pflegen die Lehnwör- 
ter aus der Sprache mit mehr Prestige zu kommen, obendrein aus den Teilen mit mehr Prestige der Lexik zu kommen, sowie ihre Konnotationen pflegen mehr Prestige zu haben.

Wenn die Änderungen von äußeren Faktoren verursacht werden, wie z. B. im Falle des Sprachkontakts, dann sind sie von einem System von außerhalb ausgelöst und auf diese Weise unabhängig vom inneren Mechanismus der Sprache. Unter ,äußeren Faktoren“ versteht man also diejenigen Kräfte, die aus der Lokalisierung und dem Gebrauch der Sprache in der Gesellschaft entstehen.

Der Kontakt zwischen zwei verschiedenen Sprachen beziehungsweise Kulturen hat als Ergebnis, dass die eine Kultur neue Sachen und Begriffe kennenlernt, und um diese benennen zu können, entlehnt man für diese Wörter aus der Sprache der anderen Kultur. Die eine Kultur kann von der anderen lernen, wobei alles von den Bedingungen des Kontakts abhängt. Im Allgemeinen findet keine Entlehnung von Wörtern statt, wenn die zwei Kulturen und ihre Sprachen nicht in Kontakt kommen. Die Richtung der Entlehnung ist gelegentlich unberechenbar. Wenn die lexikalische Entlehnung stattfindet, ist dies nicht zufällig in dem Sinne, dass ihre Richtung und Quantität von einer Sprache zur anderen variieren, oft abhängig vom Grad der kulturellen, ökonomischen und militärischen Dominanz.

Im 13. Jahrhundert gerieten die drei skandinavischen Sprachen, Dänisch, Norwegisch und Schwedisch, unter den starken Einfluss des Niederdeutschen. Das Niederdeutsche (oder das Plattdeutsche) wurde von den hanseatischen Kolonnisten gesprochen, die sich in großer Anzahl in bedeutenden skandinavischen Handelsstädten wie Bergen in Norwegen und Visby in Schweden niedergelassen haben. In jener Zeit übernahm der Hansabund die Macht über den Handel und das wirtschaftliche Leben in Nord-Europa. Diese Entwicklung setzte sich in den folgenden Jahrhunderten fort: die Hanse gewann stets an Bedeutung und in kurzer Zeit wurde sie zu einer Großmacht.

Auf norddeutschem Gebiet, dem Herrschaftsgebiet der Hanse, entwickelte sich die niederdeutsche (eigentlich die mittelniederdeutsche) Schriftsprache, die eine wichtige Rolle auch im ganzen Ostseeraum und Skandinavien spielen sollte. Mit ihren Grundlagen in den norddeutschen Städten Bremen, Hamburg und insbesondere Lübeck, wurde sie zur Handelssprache, zur Geschäft- und Verkehrssprache der Hanse, zu einer Art lingua franca, aber auch zur Prestigesprache in Nord-Europa.

Die norddeutschen Hanseaten unternahmen Handelsreisen und gründeten große und starke Kaufmannskolonien. Die Art und Weise der norddeutschen Kaufleute, ihre Lebensführung galt als Vorbild, das von den Einheimischen unbedingt nachgeahmt werden sollte und wurde. Durch den täglichen Umgang mit den Deutschen übernahmen die Skandinavier Alltagswörter wie arbejde, bruge, håb, lære, skænke, tænke, ærlig aber auch eine Menge Kulturwörter, die neue Begriffe, Gegenstände und Tätigkeiten bezeichneten (mast, spyle, borger, borg- 
mester, betale, billig, handel, købmand, lærling, pris, rådhus, skomager, køkken, frokost, kniv, gaffel, måltid, krydder, eddike).

Die Entlehnung der sogenannten Kulturwörter ist ein solcher Vorgang, der derzeit stattfindet, wenn man neue Kulturerscheinungen, neue Begriffe und Gegenstände benennt, die aus einem anderen Land oder einer anderen Kultur übernommen werden. Die niederdeutschen Wörter waren notwendig für die skandinavischen Sprachen, und sie trugen zur Erweiterung des Wortschatzes der drei skandinavischen Sprachen bei.

Das Potential für die sprachliche Änderung kann zwar von internen Faktoren kommen, aber die äußeren oder außersprachlichen Faktoren sind diejenigen, die entscheiden, ob sich die Neuerungen durchsetzen werden. Außerdem sind die äußeren Faktoren entscheidend bezüglich das Überleben der Lehnwörter. Die äuBeren Faktoren bestimmen nicht nur was überlebt, welche von den Formen die von außerhalb der Sprache kommen, sondern auch was wird überleben von den Neuerungen innerhalb der Sprache. Je stärker ist die soziale Unterstützung, die die Neuerungen und die Lehnwörter bekommen, desto größer sind die Chancen, dass sie überleben. Das bedeutet, dass es einen Druck gibt, der von den äußeren sozialen Faktoren aus in die Richtung innere Faktoren geht. Die Neuerungen müssen eine soziale Unterstützung haben, während die Lehnwörter eine soziale oder eine soziale und eine linguistische Unterstützung. Die sprachliche Änderung ist also eine Änderung der sozialen und sprachlichen Normen, während die inneren Faktoren strukturelle Einschränkungen setzen für die Neuerungen und die Lehnwörter, die sich in einer Sprachgemeinschaft verbreiten können.

Im Kontakt zwischen dem Niederdeutschen und den skandinavischen Sprachen in der Hansazeit wurden niederdeutsche Wörter aus jedem Lebensbereich aufgenommen, selbst wenn es in manchen Fällen in den skandinavischen Sprachen Wörter mit derselben Bedeutung gab. Die Prestige des Niederdeutschen führte nicht nur zur Entlehnung solcher Wörter, die keine skandinavische Entsprechung hatten, sondern auch zur Ablösung existierender skandinavischer Wörter. In einigen Fällen gelang es den einheimischen Wörtern neben den neu eingeführten zu überleben, so z. B. nytte und bruge, strid und kamp, æt und slægt, manchmal kam es zu semantischen Differenzierungen (z. B. lege und spil$l e$, oder ild und fyr, lede (efter) und søge), in anderen Fällen aber ersetzten die neuen Wörter die alten einheimischen, wie z. B. arbejde, betale, frihed anstatt erfiđi, gjalda, frjálsi. (In den obgenannten Beispielen, sowie in den folgenden werden die dänischen Formen vorgeführt).

In manchen Fällen fand nur eine semantische Beeinflussung statt. Im Altskandinavischen bedeutete dum 'schwerhörig', die Bedeutung 'dumm' ist auf den deutschen Einfluss zurückzuführen; ebenso die Bedeutung 'meinen' von finde 'finden'.

Nicht nur Wörter, sondern auch Wortbildungselemente wurden übernommen, meistens direkt mit dem niederdeutschen Wortstamm. Manchmal fügte man die 
Affixe zu den skandinavischen Stämmen hinzu und leitete neue Wörter ab. Die wichtigsten niederdeutschen Präfixe sind an- (angreb, anlæg), be- (begå, begynde, bestemme, besøg), bi- (bistand, bifald), for- (fordærve, forlade, fortjene), ge(gevær), und- (undgå). Suffixe niederdeutscher Herkunft sind -agtig (rundagtig), -bar (frugtbar, strafbar), -ere (studere), -eri (bageri, trykkeri), -else (begravelse, følelse), -hed (frihed, svaghed), -inde (fyrstinde, grevinde), -ske (syerske). Diese Wortbildungselemente wurden angeeignet, assimiliert, sie wurden Teil der skandinavischen Sprachen.

Wichtig ist auch die Entlehnung von Satzbau- und Wortbildungselementen. Niederdeutsche Affixe waren dazu berufen, in der weiteren Entwicklung der skandinavischen Sprachen eine zentrale Rolle zu spielen. Präfixe wie an- und bewurden immer zusammen mit einem anderen niederdeutschen Element, einem niederdeutschen Wortstamm entlehnt. Sie sind heute nicht mehr produktiv, im Gegensatz zu -hed / -het und -bar, die zu einheimischen Handlungsverben bzw. einigen Adjektiven hinzugefügt worden und zur Entstehung von Ableitungen wie farbar, flytbar, renhed, træthed führten. Es entstanden auch Hybridbildungen, aus einem einheimischen Wortstamm und einem deutschen Wortbildungselement, das nach dem deutschen Vorbild angenommen wurde: forelske sig 'sich verlieben', forstå 'verstehen', antage 'annehmen'.

Der Kontakt mit dem Niederdeutschen spielte eine bedeutende Rolle auch im Zerfall des reichen morphologischen Systems und in der Entwicklung einer mehr analytischen Sprache, mit dem beinahe vollständigen Verlust der Flexionsendungen. Die Tendenz, die zur Ersetzung der Flexionsformen durch Periphrasen und Wortstellungskonvention geführt hat, hatte notwendigerweise zur Folge eine beträchtliche Entwicklung im Gebrauch der Präpositionen und eine Spezialisierung ihrer Funktionen. Man kann sagen, dass durch die Ersetzung des alten Flexionssystems die Wortstellung die strukturelle Funktion, die das Wort gehabt hatte, übernommen hat.

Leonard Bloomfield unternimmt in seinem Buch Language (1967[1935]) eine Einteilung der Entlehnungstypen in kulturelle und dialektale Entlehnungen, wobei die kulturellen Entlehnungen noch in allgemeine und intime Entlehnungen unterteilt werden können.

Kulturelle Entlehnungen erscheinen im Kontakt zwischen zwei Sprachen, die gesprochen werden ,,in what it is topographically and politically a single community" (in dem, was topographisch und politisch eine einzige Gemeinschaft ausmacht) (Bloomfield 1967: 461). Die allgemeinen kulturellen Entlehnungen sind in den meisten Fällen lexikalische Entlehnungen. Solange die beiden in den Kontakt einbezogenen Teile einander etwas zu bieten haben, gehen die entlehnten Wörter in beide Richtungen. Die allgemeinen kulturellen Entlehnungen werden gewöhnlich von der Empfängersprache vollständig assimiliert, sowohl phonologisch als auch morphologisch. Eine große Menge Entlehnungen und ein langwieriger Kon- 
takt können als Ergebnis auch strukturelle Änderungen in der Empfängersprache haben, obendrein auch neue Laute und Lautgruppen einführen.

Im Gegensatz zur allgemeinen kulturellen Entlehnung ist die intime Entlehnung einseitig, sie findet nur in eine einzige Richtung statt. Diese Art von Entlehnung beschränkt sich nicht nur auf neue Begriffe, setzt die Existenz einer dominanten und einer dominierten Sprache, eines Superstratums und eines Substratums voraus. Die Wörter werden aus der dominanten in die dominierte, untergeordnete Sprache, besser gesagt in die Sprache der dominierten, untergeordneten Volksgruppe entlehnt. Die dominierte Volksgruppe ist politisch, sozial, zahlmäßig, kulturell und einschließlich sprachlich unterlegen, so dass oft die wesentliche Ursache für die linguistische Entlehnung die mit der überlegenen Gruppierung assoziierte Prestige ist. In den meisten Fällen ist die dominierte Sprache die einheimische, und die dominante die der Eroberer. Oft, nach einer Periode der Zweisprachigkeit, verzichtet die unterlegene Gruppe auf ihre Sprache zugunsten der dominanten Sprache. Als Beispiel für eine Kontaktsituation mit intimen Entlehnungen gibt Bloomfield die französisch-normannischen Lehnwörter im Englischen.

Wie im Falle der allgemeinen kulturellen Entlehnungen ist auch hier die Rede meistens von lexikalischen Entlehnungen, aber die Anzahl dieser Lehnwörter ist viel größer, was Konsequenzen auch auf die Phonologie und Morphologie der Empfängersprache haben kann.

Der Mangel an Prestige ist oft neben dem geringeren Anzahl der Sprecher gegenüber der konkurrierenden Sprache ein entscheidender Grund des Sprachwechsels, des Übergangs von einer Sprache zur anderen. Der Kontakt mit der dominanten Bevölkerungsgruppe erzwingt aus sozio-ökonomischen Gründen, dass Angehörigen der Minderheitssprache die Sprache der Mehrheit verwenden. Deswegen engt sich der Verwendungsbereich der Minderheitssprache mehr und mehr ein, und beschränkt sich nur auf den Gebrauch in der Familie und in einem kleineren Kreis. Angesichts der beschränkten Anwendungsmöglichkeiten, wird die Sprache weniger attraktiv für die neuen Generationen, und die Eltern hören auf, die Sprache mit ihren Kindern zu sprechen. Die Gleichgültigkeit, die Ignoranz der Minderheitsgruppe kann den Prozess des Sprachwechsels, den Übergang zu einer anderen Sprache beschleunigen. Obwohl die Mitglieder der Gemeinschaft eine starke Verbindung zur Sprache ihrer Vorahnen fühlen, übertragen sie sie nicht ihren Kindern. Die Folgen sind dann folgende: die Sprache wird nicht mehr gesprochen, und so wird auch ihre Existenz bedroht. Außer der Prestige und der relativen Anzahl hängt das Schicksal einer Sprache auch von anderen Faktoren ab, wie zum Beispiel dem Verhältnis ihrer Sprecher der Sprache gegenüber, der Motivation, sie zu behalten, dann noch, ob es eine Literatur gibt, ob die Sprache eine Standardvariante ist oder nicht, ob sie zu einer Religion verknüpft ist usw., aber der Prestigemangel und die geringe Anzahl der Sprecher sind die wichtigsten Faktoren. 
Die Prestige ist der wichtigste Faktor, derjenige der bestimmt, welche Wörter und Strukturen infolge des Sprachkontakts entlehnt werden. Der Mangel an Prestige kann in extremen Fällen zum Verzicht auf eine Sprache und zum Übergang zu einer anderen Sprache führen.

\section{Literaturverzeichnis}

Appel, René-Muysken, Peter 1987. Language Contact and Bilingualism. London, Edward Arnold.

Baetens Beardsmore, Hugo. 1986. Bilingualism: Basic Principles. Clevedon, Multilingual Matters Ltd.

Blake, Norman (ed.) 1992. The Cambridge History of the English Language, vol. II: 1066-1476. Cambridge, Cambridge University Press.

Braunmüller, Kurt 1995. Beiträge zur skandinavischen Linguistik. Oslo, Novus Forlag.

Breivik, Leiv Egil-Jahr, Ernst Håkon (eds.) 1989. Language Change. Contributions to the Study of Its Causes. Berlin-New York, Mouton de Gruyter.

Chomsky, Noam 1965. Aspects of the Theory of Syntax. Cambridge-Massachusetts, MIT Press (Special Technical Report No. 11)

Comrie, Bernard 1981. Language Universals and Linguistic Typology. Oxford, Basil Blackwell.

Crystal, David 1987. The Cambridge Encyclopedia of the Language. Cambridge, Cambridge University Press.

Denison, David 1993. English Historical Syntax. London-New York, Longman.

Fennell, Barbara A. 2001. A History of English. A Sociolinguistic Approach. Oxford-New York, Blackwell Publishers.

Fischer, Olga 1992. „Syntax“, in: N. Blake (ed.): The Cambridge history of the English language, Vol. II. Cambridge, Cambridge University Press, 207-408.

Fischer, Olga-Van Kemenade, Ans-Koopman, Willem-van der Wurff, Wim 2000. The Syntax of Early English. Cambridge, Cambridge University Press.

Gerritsen, Marinel-Stein, Dieter (eds.) 1992. Internal and External Factors in Syntactic Change. Berlin-New York, Mouton de Gruyter.

Greenberg, Joseph H. (ed.) 1966. Universals of the Language. 2nd Edition. Cambridge-Massachusetts, MIT Press.

Harris, Alice-Campbell, Lyle 1995. Historical Syntax in Cross-linguistic Perspective. Cambridge, Cambridge University Press.

Hock, Hans Henrich 1991. Principles of Historical Linguistics. $2^{\text {nd }}$ Edition. Berlin-New York, Mouton de Gruyter. 
Hock, Hans Henrich-Joseph, Brian D. 1996. Language History, Language Change, and Language Relationship. An Introduction to Historical And Comparative Linguistics. Berlin-New York, Mouton de Gruyter.

Jakobson, Roman. 1962. Selected Writings, Vol. I, Principles de phonologie historique. The Hague, Mouton de Gruyter.

Jeffers, Robert J.-Lehiste, Ilse 1979. Principles and Methods for Historical Linguistics. Cambridge, Massachusetts-London, England, The MIT Press.

Jespersen, Otto 1968 [1938]. Growth and Structure of the English Language. New York-London, Collier-Macmillan Limited, The Free Press.

Jespersen, Otto 1922. Language. Its nature, development and origin. London, George Allen-Unwin.

Kotsinas, Ulla-Britt-Helgander, John (utg.). 1994. Dialektkontakt, språkkontakt och språkförandring $i$ Norden. Föredrag från ett forskarsymposium, Stockholm.

Labov, William 1972. Sociolinguistic Patterns. Philadelphia, University of Pennsylvania Press.

Labov, William 1994. Principles of Linguistic Change, volume I: Internal Factors. Oxford, Blackwell.

Lehmann, Christian 1992. Word order change by grammaticalization, in: Gerritsen, Marinel-Stein, Dieter (eds.): Internal and external factors in syntactic change. Berlin-New York: Mouton de Gruyter (Trends in Linguistics, 61), 395-416.

Mallinson, Graham-Blake, Barry J. 1981: Language Typology. Cross Linguistic Studies in Syntax. Amsterdam-New York-Oxford, North-Holland Publishing Company.

Mithun, Marianne 1992: External Triggers and Internal Guidance in Syntactic Development: Coordinating Conjunctions, in: Gerritsen, Marinel-Stein, Dieter (eds.): Internal and External Factors in Syntactic Change. Berlin-New York, Mouton de Gruyter.

Paul, Hermann. 1920. Prinzipien der Sprachgeschichte. Halle, Max Niemeyer.

Romaine, Suzanne 1982. Socio-Historical Linguistics. Cambridge, Cambridge University Press.

Samuels, R. Michael 1972. Linguistic Evolution with Special Reference to English. Cambridge, Cambridge University Press.

Sapir, Edvard: Language 1970. An Introduction to the Study of Speech. London, A Harvest Book. Rupert Hart-Davis. (New York, Harcourt, Brace \& Co, 1921) Strang, Barbara M. H. 1970. A History of English. London, Methuen \& Co Ltd. Thomason, Sara Grey-Kaufman, Terrence 1988. Language Contact, Creolization, and Genetic Linguistics. Berkeley-Los Angeles-London, University of California Press. 
Torp, Arne-Vikør, Lars S. 1993. Hovuddrag i norsk språkhistorie. Oslo, Ad Notam Gyldendal.

Trudgill, Peter 1986. Dialects in Contact. Oxford, Basil Blackwell.

Trudgill, Peter 1994. 'Language Contact and Dialect Contact in Linguistic Change, in: Kotsinas, Ulla-Britt-Helgander, John (utg.): Dialektkontakt, språkkontakt och språkförandring i Norden. Stockholm, Föredrag från ett forskarsymposium.

Vikør, Lars S. 1993. The Nordic Languages. Their Status and Interrelations. Oslo, Novus Press.

Weinreich, Uriel 1953. Languages in Contact. Findings and Problems. New York, Linguistic Circle of New York. 